\title{
EFECTOS DE UN PROGRAMA DE HABILIDADES BÁSICAS EN EL DESARROLLO SOCIAL DE NIÑOS DIAGNOSTICADOS CON TRASTORNO DEL LENGUAJE EXPRESIVO - COMPRENSIVO DE TIPO DISFÁSICO PERTENECIENTES A UNA CONDICIÓN SOCIOECONÓMICA BAJA
}

\author{
Alejandro S. Dioses Chocano
}

\begin{abstract}
RESUMEN
En el presente estudio se elaboró y ejecutó un programa destinado a incrementar las habilidades básicas de 14 niños diagnosticados con tras tomo del lenguaje expresivo comprensivo de tipo disfásico, atendidos en la Clínica de Proyección Social del Centro Peruano de Audición, Lenguaje y Aprendizaje. El mencionado programa incluyó las áreas de atención, seguimiento de instrucciones e imitación.

Se efectuaron tres evaluaciones con la Escala de Madurez Social de Vineland, las mismas que permitieron apreciar un incremento en la puntuación global del desarrollo social de cada uno de los participantes, las mismas que sin embargo, no fueron significativas al analizarse la edad social y la categoría correspondiente.

Se estima que un trabajo de mayor tiempo permitiría obtener mejores, logros en las habilidades trabajadas en razón de la tendencia observada, del mismo modo, se ha generado un impacto considerables en el mejoramiento de la calidad de vida de los niños y sus familias, el mismo que se evidencia en un notable mejoramiento de las relaciones entre estos niños y sus respectivos padres.
\end{abstract}

Palabras Clave: Habilidades Básicas, Atención, Seguimiento de instrucciones, Imitación, Disfasia

\begin{abstract}
Presently study was elaborated and it executed a program dedicated to increase the basic abilities of 14 children diagnosed with dysfunction of the expressive language understanding of dysphasic type, assisted in the Clinic of Social Projection of the Peruvian Center of Audition, Language and Learning. The one mentioned program included the areas of attention, pursuit of instructions and imitation.

Three evaluations were made with the Scale of Social Maturity of Vineland, the same ones that allowed to appreciate an increment in the global punctuation of the social development of each one of the participants, the same ones that however, they didn't go significant when being analysed the social age and the corresponding category.

Is considered that a work of more time would allow to obtain better, achievements in the abilities worked in reason of the observed tendency, in the same way, a considerable impact has been generated in the improvement of the qua lit y o[the children's life and its families, the same one that is evidenced in a remarkable improvement of the relationships bellveen these children and its respective parents,
\end{abstract}

Key Words: Basic abilities, Attention, Pursuit of instructions, Imitation, dysphasic.

Gloria Díaz Acosta, María Matalinares Calvet, Kelly Falcón Díaz, Sandra Jaime, Mónica Alcántara Pérez, Sonia Acevedo León, Fanny Monge Teramae, Daniel Wilson Trujillo. 
La existencia de la patología específica en la adquisición del lenguaje denominada disfasia se reconoce desde hace ya muchos años, Narbona (1997) señala que William Wilde, en el año 1853, describió a niños que, sin ser sordos, ni tampoco retrasados mentales o paralíticos cerebrales, eran incapaces de aprender a hablar, asimismo precisa que Ajuriaguerra, en 1958, plantea la denominación de «audiomudez» para referirse a estos casos, retornando, el mismo autor, hacia 1965, el término «disfasia» para casos aparentemente menos graves. Actualmente este término es muy difundido en habla hispana, sin embargo es poco usado en lengua inglesa prefiriendo estos autores, usar las expresiones specific language impairment o specific language disorder, que se traduce como Trastorno Especifico del Desarrollo del Lenguaje o Trastorno del lenguaje Expresivo-Comprensivo.

Esta gran variabilidad de nomenclatura está asociada a otras dificultades que hacen difícil el manejo de orden terapéutico, entre estas destacan, lo heterogéneo que resulta referirse a su sintomatología, y lo aún impreciso de su etiología y mecanismos, tanto neuropsicológicos como lingüísticos.

Sin embargo, a pesar de todo lo anteriormente señalado, es factible definir un encuadre nosológico que permita definir los sujetos que conforman esta población, por ello, se manejará una definición por exclusión, asumiendo, como lo plantean la mayor parte de autores, que la disfasia o trastorno específico del desarrollo del lenguaje es «todo inicio retrasado y todo desarrollo enlentecido del lenguaje que no pueda ser puesto en relación con un déficit sensorial (auditivo) o motor, ni con deficiencia mental, ni con trastornos psicopatológicos, ni con privación socioafectiva, ni con lesiones o disfunciones cerebrales evidentes» (Rapin y cols. 1992, American Psychiatric Associaton: DSM -IV 1994, ed. Española 1995).

Consecuencia directa de lo detallado hasta el momento, es la falta de estrategias especialmente diseñadas para la educación y habilitación de estos niños, tanto en los aspectos cognitivos, como en los sociales y afectivos, pudiéndose detectar escasez de estudios sobre el particular. Sin embargo, existen autores (Galindo, 1981; Graziano 1982; Ross 1985; Fernández, 1995; Ribes, 1995) que, haciendo uso de técnicas de modificación de conducta derivadas del Análisis Conductual Aplicado, describen trabajos para desarrollar diversos tipos de repertorios de conducta en niños que, a pesar de pertenecer a categorías diagnósticas diferentes -retardo mental, autismo, parálisis cerebral infantil o sorderaevidencian comportamientos semejantes en los aspectos cognitivos y socioemocionales, reportando avances significativos en el desarrollo de sus conductas.

En todos estos estudios se destaca la importancia de trabajar primero los repertorios de Habilidades Básicas: Atención, Imitación y Seguimiento de Instrucciones como ejes fundamentales de cualquier otro desarrollo de conductas.

Un trabajo, asumiendo esta estrategia de intervención, implica la formulación de un programa que considere, como mínimo, tres aspectos: Acciones, Estructura y Temporalización (Femández-Ballesteros, 1996); además de seguirse una serie de pasos sistemáticamente estructurados: a) Análisis o evaluación de las necesidades; b) Programación, que incluye plantear los objetivos instruccionales, procedimientos de evaluación y actividades a ejecutarse; c) Implementación; y d) Toma de decisiones (Sanz, 1996).

Por ello, considerando todo lo anteriormente descrito es que se estimó pertinente plantear como problema a investigar: ¿Cuáles serán los efectos de un programa de habilidades básicas en el desarrollo social de niños diagnosticados con trastorno del lenguaje expresivo - comprensivo de tipo disfásico pertenecientes a una condición socioeconómica baja? Así planteado el problema, se propuso la siguiente hipótesis: 
H1: El desarrollo social de niños diagnosticados con trastorno del lenguaje expresivo comprensivo de tipo disfásico, mejorará significativa-mente luego de participar en un Programa de Habilidades Básicas sustentado en principios del análisis conductual aplicado.

Para esto se precisaron los siguientes objetivos:

- Diseñar un Programa de Habilidades Básicas para niños con trastorno del lenguaje expresivo-comprensivo de tipo disfásico sustentado en principios del análisis conductual aplicado.

- Incrementar el desarrollo social de niños diagnosticados con trastorno del lenguaje expresivo-comprensivo de tipo disfásico a través de la administración de un Programa de Habilidades Básicas sustentado en principios del análisis conductual aplicado.

\section{MÉTODO}

Sujetos

Los sujetos del presente estudio estuvieron conformados por 14 niños diagnosticados, de acuerdo a los criterios del CIE-10 Y del DSM-IV, con trastorno específico del desarrollo del lenguaje expresivo-comprensivo de tipo disfásico, el repertorio de lenguaje oral expresivo corresponde al de niños entre los 24 meses y 36 meses estando principalmente compuesto de estructuras denominadas palabra-frase y palabra yuxtapuesta.

Todos los sujetos son peruanos, residentes en la provincia de Lima desde el nacimiento y no tienen escolaridad. Sus padres hablan español, son alfabetos y pertenecen a una condición socioeconómica baja. Las edades de los niños fluctúan entre los 3 años y 7 años, no presentando compromisos neurológicos estructurales. El grupo experimental ha sido atendido en terapia del lenguaje por la Clínica de Proyección Social del Centro Peruano de Audición, Lenguaje y Aprendizaje en forma ambulatoria desde Agosto del año 1999.

\section{Instrumento}

\section{Ficha Técnica}

Denominación : Escala de Madurez Social de Vinneland

Autor : Edgard Doll

Año : 1925

Adaptación al español : Noemí Morales

Significación : Evaluar la capacidad social, autosuficiencia, actividades ocupacionales, comunicación, participación social y libertad para la supervisión.

\section{Descripción del Instrumento}

La prueba consta de un manual que contiene las normas de administración de la escala y un protocolo para el registro de respuestas.

Presenta 8 áreas de evaluación: Autoayuda General, Autoayuda para Comer, Autoayuda para Vestirse, Dirección de Sí Mismo, Locomoción, Ocupación, Comunicación y Socialización. 
Los ítems están distribuidos por rango de edad desde el año hasta los veinticinco años.

\section{Administración}

Se administra mediante una entrevista a los padres o personas que más conocen al niño. Se inicia la administración dos años antes de la edad cronológica y se suspende la administración en el nivel de edad donde la informante refiere que el niño fracasa en todos los ítems.

\section{Calificación e Interpretación}

Cada respuesta positiva se valora con 1 punto, las respuestas negativas con 0 puntos y las conductas medianamente logradas con $1 / 2$ punto.

Terminada la administración se obtiene el puntaje directo sumando las valoraciones obtenidas, con dicha sumatoria se obtiene la edad mental y luego, mediante una proporción, el cociente y categoría social respectiva.

\section{Procedimiento}

Se conformó un grupo de estudio mediante un muestreo no probabilístico intencionado, de la totalidad de pacientes que asistieron a la Clínica de Proyección Social del CPAL solicitando atención por dificultades de lenguaje entre los meses de Julio de 1999 y Enero del 2000.

Todos los niños seleccionados fueron sometidos a un examen clínico- neurológico para confirmar el diagnóstico inicial y posteriormente, a un proceso de evaluación psicológica, el mismo que incluyó la elaboración de la historia clínica, observación de conducta en sesiones de juego y de interacción con padres.

Posteriormente se efectuó la evaluación del Desarrollo Social mediante la administración del instrumento de investigación. Paralelamente se diseñó el Programa de Habilidades Básicas que incluyó los repertorios de Atención, Imitación y Seguimiento de Instrucciones basado en los principios del Análisis Conductual Aplicado.

Los niños seleccionados participaron diariamente, de lunes a viernes, en sesiones individuales correspondientes al Programa de Habilidades Básicas entre los meses de Mayo y Noviembre del año 2000, cada una de las cuales tuvo una duración de 15 minutos. Se efectuaron tres mediciones, antes del inicio del programa, durante el desarrollo del mismo, y al término del trabajo.

\section{RESULTADOS}

La ejecución de la investigación permitió alcanzar el objetivo de diseñar un Programa de Habilidades Básicas para niños con trastornos del lenguaje expresivo - comprensivo de tipo disfásico sustentado en los principios del análisis conductual aplicado, el mismo que constó de tres áreas de trabajo: Atención, Imitación y Seguimiento de Instrucciones (Anexo 1).

Cada una de las áreas se dividió en diferentes habilidades, y a su vez, para cada una de estas, se efectuó el análisis de los pasos secuencialmente necesarios para lograr adquirirla. La administración del programa permitió, a su vez, alcanzar el objetivo de incrementar el desarrollo social de estos niños, apreciándose que las puntuaciones totales alcanzadas en las mediciones del desarrollo social (Tabla 1), son significativamente mejores entre la primera y la tercera de las mediciones $(\mathrm{t}=-2.96)$, así como entre la segunda y la tercera de las evaluaciones $(\mathrm{t}=-2.85)$. Cuando se efectúa la comparación entre la evaluación de entrada y la segunda evaluación, la diferencia no es significativa $(\mathrm{t}=-0.50)$, esto podría deberse a que el programa aún se encontraba en su primera fase de administración, y tanto los niños, como los instructores, recién estaban adecuándose al sistema de trabajo. 
Tabla 1

Comparación de las puntuaciones totales alcanzadas por los sujetos en las tres mediciones efectuadas

\begin{tabular}{|c|l|l|c|c|c|c|}
\cline { 2 - 7 } \multicolumn{1}{c|}{} & Media & DS & $\mathbf{N}$ & $\mathbf{T}$ & gl & P \\
\hline Total 1 & 50.78 & 12.68 & 9 & & & \\
Total 2 & 51.61 & 10.61 & 9 & -0.50 & 8 & 0.631 \\
\hline Total 1 & 50.56 & 12.67 & 9 & & & \\
Total 3 & 55.11 & 9.63 & 9 & -2.96 & 8 & $0.018 *$ \\
\hline Total 2 & 53.23 & 9.24 & 11 & & & \\
Total 3 & 56.09 & 9.63 & 11 & -2.85 & 10 & $0.017 *$ \\
\hline
\end{tabular}

Las diferencias descritas anteriormente no se reflejan en las Edades Sociales obtenidas por los alumnos (Tabla 2), ya que si bien es cierto que estas mejoraron, no lo hicieron lo suficiente como establecer diferencias significativas, aunque la tendencia muestra que existía un sentido positivo en las mismas.

Tabla 2

Comparación de la edad social alcanzada por los sujetos en las tres mediciones efectuadas

\begin{tabular}{|c|c|c|c|c|c|c|}
\cline { 2 - 7 } \multicolumn{1}{c|}{} & Media & DS & N & T & gl & P \\
\hline ES 1 & 47.51 & 25.02 & 11 & -0.80 & 10 & 0.445 \\
ES 2 & 51.64 & 20.04 & 11 & & & \\
\hline ES 1 & 47.16 & 26.36 & 10 & -2.38 & 9 & 0.041 \\
ES 3 & 59.10 & 18.78 & 10 & & & \\
\hline ES 2 & 54.23 & 17.77 & 13 & -3.31 & 12 & 0.006 \\
ES3 & 60.93 & 18.78 & 13 & & & \\
\hline
\end{tabular}

Esto mismo ocurre al compararse los cocientes sociales en las diferentes evaluaciones (Tabla 3), apreciándose que, si bien es cierto se presentan cambios favorables luego de la administración del programa, dichos cambios no son significativos, es importante señalar que aquí influye la forma en que se obtiene el valor del cociente social, ya que es necesario considerarse la edad cronológica, habiéndose observado que durante el periodo de trabajo, que la edad cronológica evoluciona más rápido que la edad social convirtiéndose, esto, en un factor que incidió, desfavorablemente, en la obtención de mejores puntuaciones al momento de ser calificados. 


\section{Tabla 23}

Comparación del cociente social alcanzada por los sujetos en las tres mediciones efectuadas

\begin{tabular}{|c|c|c|c|c|c|c|}
\cline { 2 - 7 } \multicolumn{1}{c|}{} & Media & DS & N & T & gl & P \\
\hline ES 1 & 47.51 & 25.02 & 11 & & 10 & 0.445 \\
ES 2 & 51.64 & 20.04 & 11 & & & \\
\hline ES 1 & 47.16 & 26.36 & 10 & -2.38 & 9 & 0.041 \\
ES 3 & 59.10 & 18.78 & 10 & & & \\
\hline ES 2 & 54.23 & 17.77 & 13 & -3.31 & 12 & 0.006 \\
ES3 & 60.93 & 18.78 & 13 & & & \\
\hline
\end{tabular}

\section{DISCUSIÓN}

El proceso de trabajo efectuado, y los resultados obtenidos, muestran que las estrategias derivadas del análisis conductual aplicado son procedimientos eficaces que permiten la adquisición de una serie de repertorios conductuales en personas severamente afectadas. En este sentido, los niños que presentan trastorno del trastorno del lenguaje expresivocomprensivo de tipo disfásico, requieren de un conjunto de habilidades básicas, tales como atención, seguimiento de instrucciones e imitación, como parte de un desarrollo social que les permitan acceder, posteriormente, a una enseñanza sistemática del lenguaje.

De igual forma, se ha podido apreciar la necesidad de que el entrenamiento en habilidades básicas, sea de mayor extensión en el tiempo, y quizá también, incluyendo varios periodos de trabajo al día, de tal manera que se pueda lograr un desempeño más sostenido por parte de los alumnos.

\section{BIBLIOGRAFIA}

Arnau, J. (1990). Diseños experimentales en Psicología y educación. México. Trillas. Azcoaga, J. (1973). Alteraciones del lenguaje en el niño. Ed. Paidos. Buenos Aires.

Femandez - Ballesteros. (1996) Manual de técnicas de modificación de conducta. Pirámide. España.

Galindo, colb. (1981). Modificación de conducta en la educación especial. Diagnóstico y programas. Ed. Trillas. México.

Kerlinger, F. (1985). Investigación del comportamiento. México. Interamericana.

Launay; Borel-Maisonny (1989). Trastornos del. lenguaje la palabra y la voz en el niño. Ed. Masson. Barcelona.

Monfort, M.; Juarez , A. (1987). El niño que habla. Ed. cepe. Madrid.

Peña Casanova, J. (1993).Manual de logopedia. Ed. Masson. Barcelona.

Ribes, E. (1975). Técnicas de modificación de conducta: Su aplicación al retardo en el desarrollo. Trillas. México. 